\title{
ENERGY OF VIRTUAL AND REAL REALITY IN POST-CRISIS "FUTURE" PROJECTS
}

\author{
Yuri Plakitkin *1 \\ ${ }^{1}$ Energy Research Institute of the Russian Academy of Sciences (ERI RAS), Moscow, Russia
}

\begin{abstract}
The questions of the search for fundamental laws of development of the world energy and economy during the period of growth in the volume of virtual reality are considered. The results of calculations are presented, indicating the probable implementation of two large technological "leaps" in the post-crisis period.
\end{abstract}

Keywords. Virtual reality, energy, technological development, energy density, time, forecasts, energy cycles

\section{Introduction}

The future development of the world energy sector is characterized by very great uncertainty. Even on the eve of the onset of the 2020 crisis associated with the spread of the COVID-19 virus, many well-known foreign and domestic agencies demonstrated such a wide range of forecasts for the future development of the world energy sector that any analyst had legitimate doubts about the existence of a scientific basis for forecasting, taking into account the impact of modern global processes of various "Nature".

A legitimate question arises: "What awaits the global energy industry: energy transition, peak consumption of oil and other traditional energy sources?" Perhaps the world expects the use of a more powerful source of energy? Dont clear. To answer these and other questions, of course, it is necessary to conduct fundamental research on the topic: "Where is our world heading."

\section{Hybrid reality and its components}

The 2020 crisis has shown that global development is moving towards the expansion of virtual reality and is increasingly becoming hybrid. During the research, several options for the development of hybrid, virtual and real reality were considered. As a result of the analysis of these options, it was found that the hybrid reality has an expanding dynamic that has a limit to expansion. With this variant of the development of hybrid reality, its constituent components will be characterized by an expansion of virtual reality (logistic dependence) and a shrinking reality (cyclical dependence) (Fig. 1).

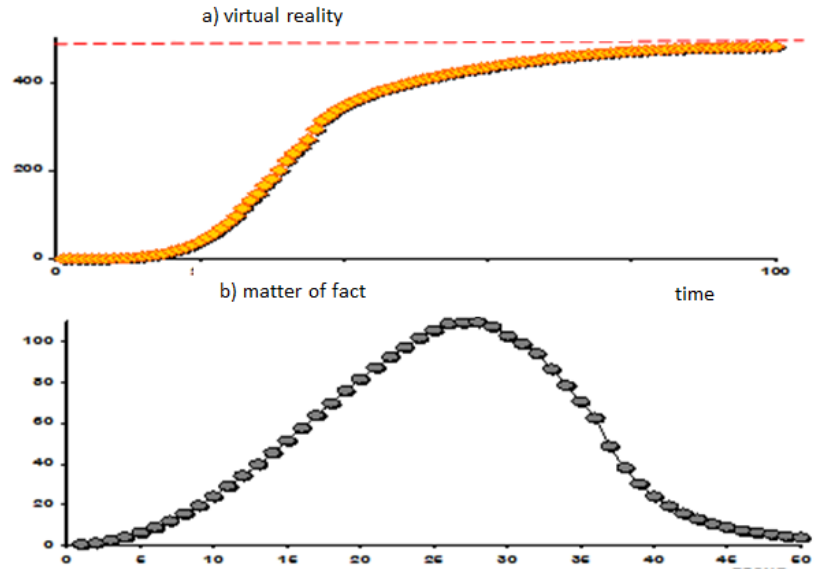

Fig. 1. Components of an "expanding" hybrid reality

The development of reality is provided by physical energy. On the basis of physical energy, work is carried out, as a result of which material results of labor appear. In general, physical energy can be calculated using the formula:

$$
E_{f}=q_{f}^{*} m
$$

where: $\mathrm{m}$ - the mass of the energy source used;

$\mathrm{q}_{\mathrm{f}}$ - physical energy density, i.e. the amount of physical energy per unit mass of the source.

In power engineering, the above given energy density is called the caloric equivalent of the fuel used. In general, the energy density of energy sources has a growth limit determined by the formula of A. Einstein:

$$
\mathrm{E}=\mathrm{m} * \mathrm{c}^{2}
$$

where: $\mathrm{c}-$ speed of light.

In this regard, the energy density of energy sources cannot be greater than $c^{2}$. Thus, the change in the energy density over time will be characterized by a logistic dependence, "abutting" the limit equal to $\mathrm{c}^{2}$. Then, in

* Corresponding author: uplak@mail.ru 
accordance with the cyclical nature of reality, the mass of the energy sources used should also change in a cyclical relationship. The development of virtual reality can be characterized by the dynamics of physical energy.

The question arises: "What characterizes the development of virtual reality?" Virtual reality is not a material space, it is a collection of knowledge, technological skills, information, etc. Given the informational nature of virtual reality, its development can be characterized by the level of entropy achieved.

However, it is known that entropy can be measured by energy:

$$
S=K^{*} L N\left(E_{v}\right)
$$

where: $\mathrm{K}$ - constant coefficient;

$\mathrm{E}_{\mathrm{v}}$ - energy of virtual reality.

The above expression indicates the possibility of evaluating virtual reality by the amount of energy used. Since virtual reality reflects the level of accumulated knowledge of technology and information, it characterizes scientific and technological development.

In this regard, the energy of virtual reality is a special type of energy - the energy of scientific and technological development.

In accordance with the universal nature of the calculated energy formulas, the energy of virtual reality can be represented as:

$$
E_{v}=q_{v}^{*} m
$$

where: $\mathrm{q}_{\mathrm{v}}$ - energy density of virtual reality;

$$
\mathrm{m}-\text { the mass in virtual reality. }
$$

The above indicates that both virtual and real reality have a single energetic "nature". At the same time, it is very important to establish the relationship between physical energy and the energy of virtual reality. In the course of the research, it was revealed that the fulfillment of such fundamental laws as the law of conservation of energy and the law of conservation of mass requires the fulfillment of the condition of equality of the density of physical energy and the energy density of virtual reality:

$$
q_{f}=q_{v}
$$

Equality (5) is of fundamental importance. It testifies that the development of virtual reality and, accordingly, the level of scientific and technological development can be measured by the density of physical energy of the energy sources used. It turns out that the caloric equivalent (energy density) of energy sources used in the economy determines the level of technological development. If in equality (5) the current energy density is related to its limiting density (c2), then we can obtain a logistic dependence of the change in the relative energy density over time. In this case, the limiting value of the relative energy density will be equal to unity (Fig. 2).

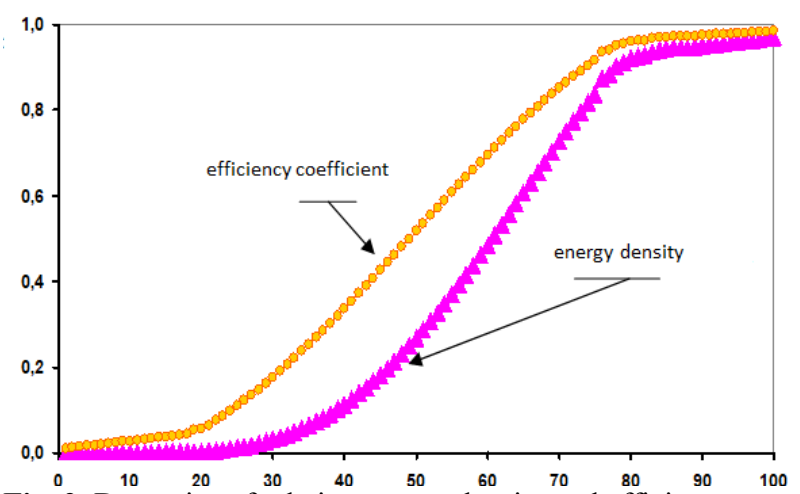

Fig. 2. Dynamics of relative energy density and efficiency

Specific energy density (see Fig. 2) has a fairly strong relationship with the efficiency of machines and mechanisms used in the economy. The change in efficiency reflects the level of technological development of the economy. This circumstance strengthens the argument that the energy density of the used energy sources can characterize the level of technological development of the economy.

\section{The Energy of the waves of technological development and time}

Analyzing the relationship between such fundamental categories as "time" and "energy", we will conduct a thought experiment: "look" into the past from the standpoint of the current present and try to find what was in common that characterized the implementation of all the processes and phenomena in the global technological development. All phenomena and processes are characterized by the same form of realization ций cyclical development, in which the stages are sequentially carried out: growth, achievement of maximum values, decline. As you get closer to the present, the cycle frequency increases. There is a more rapid change in technologies and applied technical solutions. This frequency determines the speed of the technological process in the global economy. Based on an expert assessment of the frequency of cycles of technological development, the forecast dynamics of the average frequency of cycles of world technological development has been established (Fig. 3). 


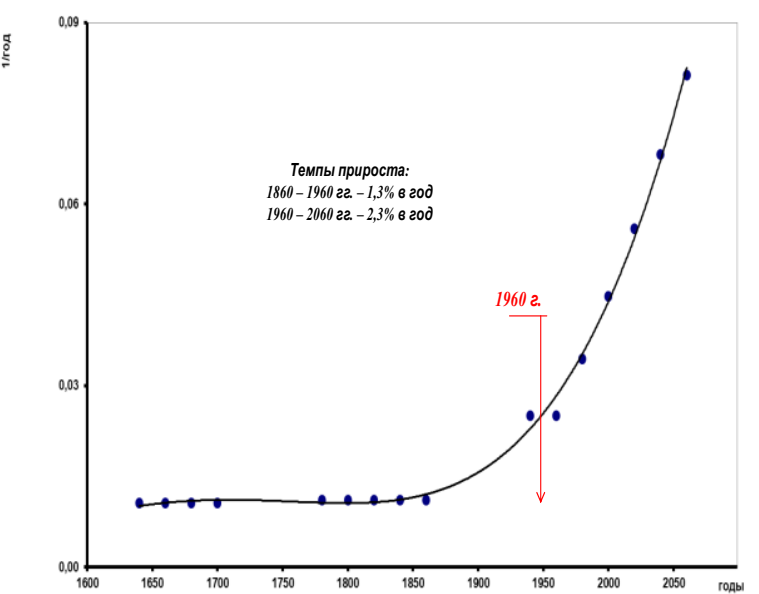

Fig. 3. Base frequencies adopted for assessing the cycles of global technological development

Since the 60 s of the XX century, there has been a significant increase in the average annual rate of increase in the frequency of cycles. The cycle frequency began to change exponentially. In accordance with this frequency, in the process of research, a model of the wave (average) of world technological development was built, which can be described by an equation of the form:

$$
Y_{t}=A * \operatorname{Sin}\left(6,28 * v_{t} * t\right)
$$

where: A - wave amplitude;

$\mathrm{V}_{\mathrm{t}}$ - wave frequency at time;

$\mathrm{t}$ - time.

The wave of world technological development has energy, the density of which can be determined by the expression:

$$
W_{t}=\frac{\rho * A_{t}^{2} *\left(6,28 * v_{t}\right)^{2}}{2}
$$

where: $p$ - density of the medium in which the wave propagates.

Note that the energy density of a wave depends on the square of its frequency. Taking into account the exponential increase in the frequency of the wave in the future period, one can state an unprecedentedly high increase in the energy density during the period of significant immersion of world development in virtual reality (Fig. 4).

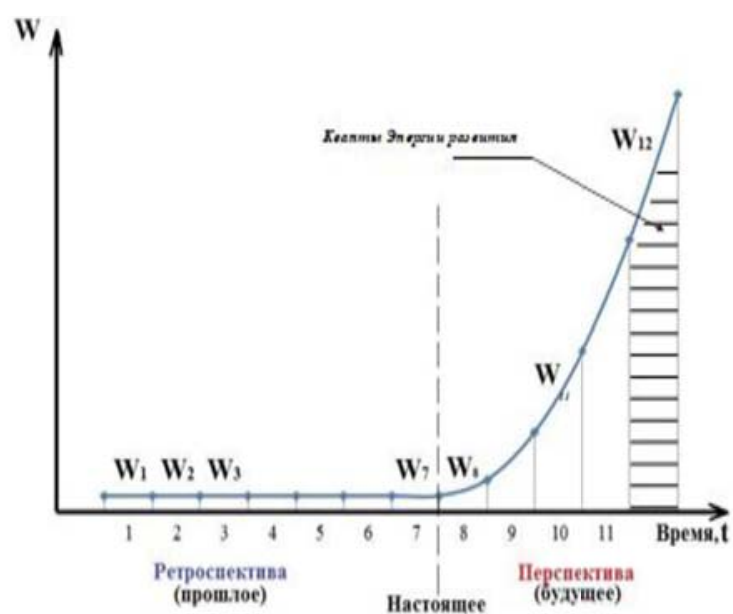

Fig. 4. Forecast corridor of dynamics of energy density (caloric equivalent) in the global energy sector of the XXI century

In fig. 4 shows that each current year is characterized by the "input" of a new quantum of energy. Moreover, the value of the "introduced" quanta significantly increases in the future period of time. In fact, physical time, which is used in world practice, is nothing more than just the ordinal number of "introduced" quanta of energy of technological development. So, what comes out all forecast calculations in the economy, energy, etc. Researchers carry out not by "the phenomenon itself", but by its ordinal number? In general, such forecasting is rather paradoxical. Yes, this was acceptable in the past period (see Fig. 4), since the introduced energy quanta were not very large, but in the promising period of high rates of energy growth in technological development, such forecasting becomes untenable.

We need approaches to forecasting based on taking into account the growth of energy of technological development. The energy of technological development is primary in relation to the emergence of new technologies. During the entire civilization process, there was a change in energy sources in the direction of a higher energy density provided by them. This was accompanied by a change in global energy development cycles. Wood, coal, oil, gas, energy cycles, replacing each other, increase the energy density (caloric equivalent) used in the world economy. Due to such an upward increase in energy density, it should be expected that the 21 st century is the period of the beginning of the use of sources with high and very high caloric equivalents. In accordance with the change of energy sources, new technologies used in the world economy appear. For example, the use of such an energy source as coal led to the creation of a steam engine, a steam locomotive, the construction of railways and stations. The beginning of this process was a new energy source, and not the other way around. It was not train stations, railways and a steam locomotive that determined the appearance of an energy source.

\section{Big technological leaps in the coming period}


Taking into account the caloric equivalents of energy sources achieved in the global energy industry, as well as the logistic nature of their change over time, in the process of research, a predicted corridor of energy density values was obtained that can be achieved in the 21 st century (fig. 5).

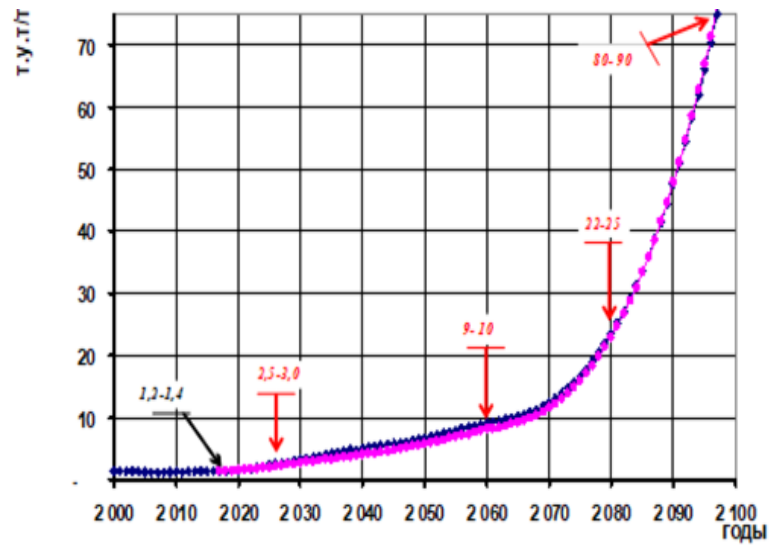

Fig. 5. Forecast corridor of dynamics of energy density (caloric equivalent) in the global energy sector of the XXI century

The resulting predictive dynamics of energy density indicates that in the next ten-year period (2020-2030), it should be doubled. By the middle of the 21 st century, the energy density, in relation to the period 2020-2030, should double for the second time, and by the end of the century its values should reach values exceeding 100 tons of fuel equivalent per ton $(t / t)$. A twofold increase in energy density in the next 10-year period, in all likelihood, means widespread use of hydrogen in the world economy.

What is the energy of the fuel used? It can be stated with all certainty that this energy is converted into the kinetic energy of the movement of machines and mechanisms that perform certain work, as a result of which new goods and services are received in society.

In the course of the study, it was found that in the nearest forecast period the average speed of movement of people and goods in the economy (implementation of kinetic energy) will be proportional to the square of the energy density of the energy sources used.

By doubling the energy density, for example, by switching from the use of methane to the use of hydrogen, it can lead to a 4-fold increase in the average speed of movement in the economy. It is clear that in the limiting case (when the speed of movement is equal to $\mathrm{c}^{2}$, the speed of movement will be determined not by the square of the energy density, but by the power factor at it equal to 0.5 .

In accordance with the predicted dynamics of the energy density values reached in the XXI century, possible levels of the speed of movement of people and goods in the world economy have been established (fig. 6).

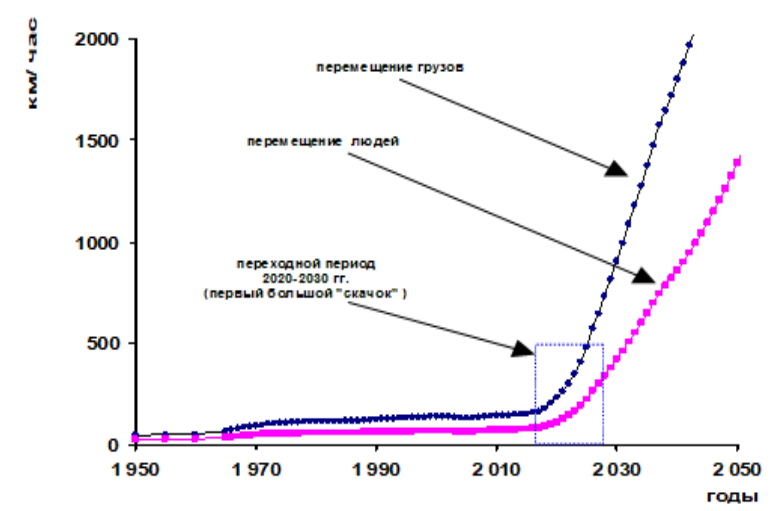

Fig. 6. Forecast dynamics of the average speed of movement of goods and people for the period up to 2050

Calculations show that already in the period 20202030. there will be a "break" in the predicted trajectory of the speed of movement in the economy during this period. This speed will receive an impulse for its further growth. It is obvious that this impulse is associated with the powerful "impact" of new technologies used in the economy.

Most likely 2020-2030 - these are the years of transition, in which the first big technological "leap" will take place. Calculations show that the second technological "leap", which, respectively, determines the second impulse to increase the average speed of movement in the economy, will be implemented approximately in 2060-2070. (fig. 7).

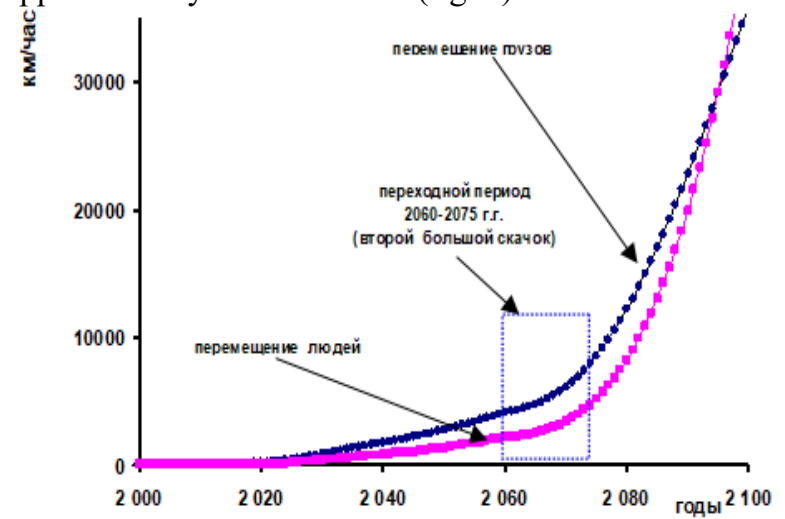

Fig. 7. Forecast assessment of the dynamics of the average speed of movement of goods and people for the period up to 2100 .

Apparently, the first big "leap" will be associated with the implementation of the world project "Industry $4.0 "$, and the second, respectively, with the project "Society 5.0".

It is significant that the leader in the implementation of the Industry 4.0 project, Germany, probably "realizing" that a technological "leap" cannot be made on the basis of the energy density of the energy sources used, in June 2020 adopted the national program "Hydrogen Energy" and started to its implementation. For this purpose, two program committees (government and scientific-public) were created to finance and promote technologies for the production and transportation of hydrogen.

The higher the speed of movement in the economy, the more work is done per unit of time and, therefore, the 
higher the labor productivity achieved in the economy. Calculations show that by the middle of the twenty-first century, one can expect that labor productivity in the economy may increase by $8-10$ times. Such an increase in labor productivity can be achieved through the use of intelligent cyber-physical systems provided for by the world project "Industry-4.0". The growth in labor productivity will lead to a decrease in the supply prices of goods and services in the world economy. In accordance with the carried out model calculations, estimates of a possible price reduction were obtained for three options for the impact of labor productivity on production costs (very weak, weak and strong impact). In all cases, a decrease in prices was obtained in the prospective period. Even if the impact is weak, supply prices are likely to be reduced by at least $15 \%$ (by 2050). The maximum price reduction can be approximately 40 $50 \%$.

\section{Forecast cycles and parameters of global energy development}

In addition to the average speed, in the economy, in the process of research, the predicted values of the maximum travel speeds were obtained. These speeds are calculated on the basis of retrospective dynamics of maximum speeds, some of which are associated with the implemented space projects. The retrospective dynamics of the above mentioned speeds, as well as their "convergence" with an average speed in a deep perspective period, made it possible to form predictive estimates of the maximum speeds.

Note that the maximum speed in the previous period of time was first associated with the achievement of the first cosmic speed, intended for the movement of the spacecraft in the Earth's orbit. Then the second cosmic speed, allowing you to leave the Earth's orbit. And, finally, the third, achieved in 2013, and allowing the spacecraft to leave the solar system.

In accordance with the calculations, it was found that approximately, in the period 2055-2060. the 4th space speed must be reached, allowing the spacecraft to leave the Galaxy.

Most likely, the main mission of Mankind at the present stage of development is the expansion of outer space. First of the near, then the middle and, finally, the far space. The implementation of such a mission provides for the implementation of large-scale space projects that "pull" projects carried out in the economy.

Predictive estimates of the maximum speeds of movement made it possible to determine the future dynamics of changes in the maximum values of the energy density of the energy sources used.

In accordance with the carried out model calculations, it was found that, approximately, in 20422047. the massive use of energy sources based on nuclear fission should begin. Most likely, these will be small-sized sources (fuel cells) that directly convert nuclear energy into energy energy.

Approximately in 2055-2060. perhaps the beginning of industrial use of the energy of thermonuclear fusion.
Such an event, of course, will have a very significant impact on all spheres of human activity. Note that in the same period it is planned to reach the fourth cosmic speed. In addition, in the above-mentioned period of time, the level of artificial intelligence will significantly increase - from the category of "strong", achieved by 2040, it will become "very" strong. "Artificial intelligence with such a level will have abstract thinking that allows them to engage in creative and managerial work.

The period of the 60 s of the XXI century, which provides for the implementation of the second big technological "leap", will be characterized by three significant events:

- the beginning of the use of energy sources based on thermo-nuclear fusion;

- the development of a very strong artificial intelligence;

- reaching the fourth cosmic speed, allowing the expansion of deep space.

The obtained predicted values of the average and maximum energy density (caloric equivalents) made it possible to carry out model calculations to identify future cycles of global energy development (Fig. 8).

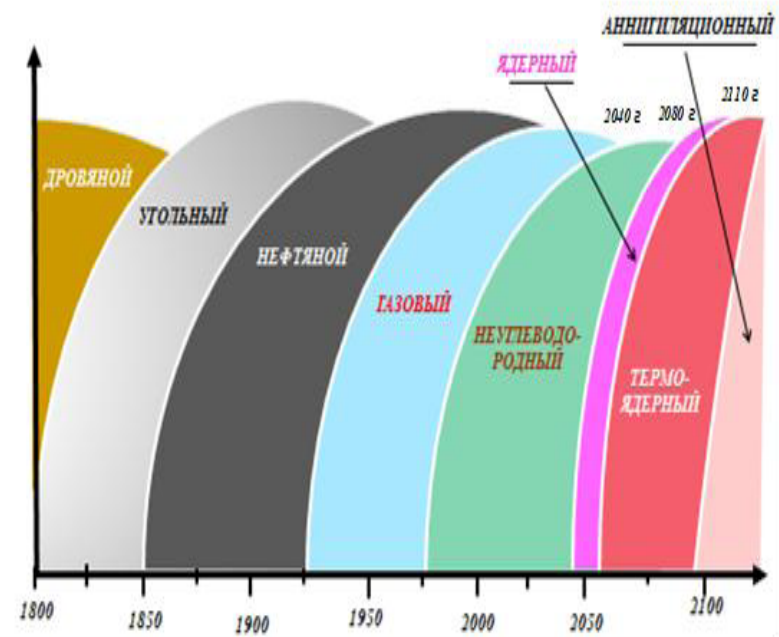

Fig. 8. Forecast cycles of global energy development

Calculations show that after the oil cycle, which in turn replaced the coal cycle, the dominance of the gas source will emerge in the near future. It will manifest itself approximately in 2035-2040, and then non-carbonhydrogen energy resources, including all the variety of solar, wind, geothermal, hydro and nuclear sources, will become the dominant energy source.

Note that the period 2020-2040. will be characterized by a wide variety of traditional and non-hydrocarbon energy sources used. The dominance of nuclear sources of direct energy conversion will come approximately in the period 2080-2100.

Outside of this period, thermonuclear energy sources will dominate, the industrial development of which will probably begin in 2055-2060.

The established cycles of global energy development made it possible to assess the forecast dynamics of 
global consumption of traditional and renewable energy sources (Fig. 9).

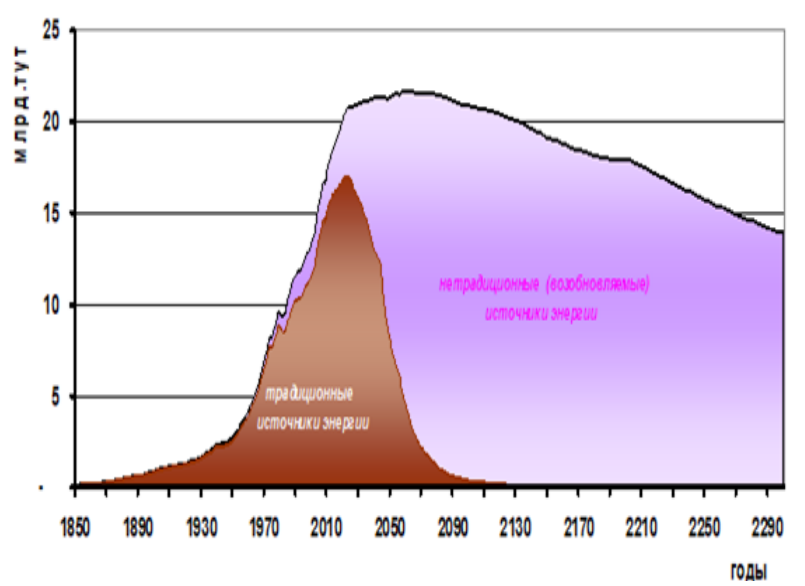

Fig. 9. Forecast dynamics of global consumption of traditional and renewable energy sources

The calculations made it possible to establish the dynamics of reducing the consumption of traditional energy sources in the coming period.

For a long time, many experts argued that energy consumption will increase in the forecast period. However, the calculations carried out not only do not confirm the presence of future "upward" trends in energy consumption, but on the contrary, they indicate a decrease in both the total world energy consumption and its per capita consumption. At the same time, the coming period of time will be characterized by a slight increase in energy consumption, turning into stabilization, approximately until the 90s of the current century.

Outside this period, there is a high probability that global energy consumption will decline. The decrease in consumption of traditional energy sources will occur against the background of a decrease in the main modern energy carriers - oil and gas. So, in accordance with the calculations, a twofold reduction in oil consumption is likely to be realized approximately in 2045-2048.

The subsequent, another twofold decrease in oil consumption, will begin approximately in the $60 \mathrm{~s}$, during the period when thermonuclear energy sources began to be used.

Gas energy sources, having reached their maximum level of consumption, are most likely to keep it until about the 40s of this century. A twofold decrease in gas consumption is likely to occur in the period 2050-2055.

The subsequent twofold decrease is likely to occur in the period 2065-2070, that is, during the period of growth in the use of thermonuclear energy sources. Such a "downward" dynamics of the use of traditional energy resources will lead to a decrease in prices for the world's main energy carrier - oil. Calculations have established that the world oil price will continue its systemic decline and by 2040 , most likely, will drop to $\$ 20-25$. US / bar. (average annual oil price). Despite the existing opinion of some experts and representatives of the state regulator that the oil price will "win back" its positions, the calculations carried out indicate its further decline.

\section{Conclusion}

The presented forecast tendencies form a multiprofile "image of the future" realized in the context of expanding virtual reality. An assessment of its parameters based on fundamental research by the author of the publication can be used to make investment and organizational decisions developed by representatives of Russian business and government bodies.

\section{Literature}

1. Bushuev V.V., Leontiev N.P. Digital society // Economic strategies. 2019.No. 6 (164). P. 96-105

2. Hillier B. Industry 4.0: Smart Production of the Future. Experiense in Digitalization in Germany / Presentation // Information Modeling for Infrastructure Projects and Business Development in Greater Eurasia: VI Intern. Forum. Moscow. 7 June, 2017.http: / 3d-couf. ru / pdf - 2017. Accessed: 04/29/2019.

3. Plakitkin Yu.A., Plakitkina L.S. The world innovative project "Industry-4.0" — possibilities of application in the coal industry of Russia. 1. Program "Industry 4.0" - new approaches and solutions // Coal. 2017. No. 10. P. 44-50. DOI: 10/18796 / 0041-5790-2017-10-44-50.

4. Noritsugu U. "Society 5.0": the view of Mitsubishi Electric // Economic strategies. 2017. No. 4.

5. Makarov A.A., Makarov A.A. Regularities of energy development - an elusive essence // Izv. AN. Energy. 2010. No. 1. P. 3-12.

6. Modeling and forecasting of world dynamics development and improvement of methods of analysis and mathematical modeling of various aspects of world dynamics / V.A. Sadovnichy, A.A. Akaev, A.V. Korotaev, S.Yu. Malkov. M .: ISPI FNISTS, 2012.

7. Eurasian energy civilization. On the issue of "energy of the future" / V.V. Bushuev A.M., Mastepanov, V.V. Pervukhin, Yu.K. Shafranik. M $\quad \therefore$ EC "Energia", 2017 - 208 p.

8. Kapitsa S.P., Kurdyumov S.P., Malinetskiy G.G. Synergetics and forecasts of the future. 4th ed., Rev. and add. M .: URSS, 2019.

9. Hawking S.W. A Brief History of Time. N.Y.: Bantam Books, 1988. - 231p. ISBN 978-5-36700490-8 